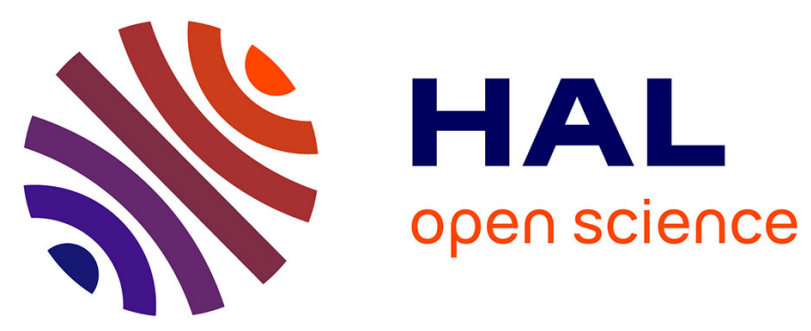

\title{
WSN4QoL: WSNs for remote patient monitoring in e-Health applications
}

S. Tennina, M. Santos, A. Mesodiakaki, P.-V. Mekikis, E. Kartsakli, A. Antonopoulos, M. Di Renzo, A. Stavridis, F. Graziosi, L. Alonso, et al.

\section{- To cite this version:}

S. Tennina, M. Santos, A. Mesodiakaki, P.-V. Mekikis, E. Kartsakli, et al.. WSN4QoL: WSNs for remote patient monitoring in e-Health applications. IEEE International Conference on Communications (ICC 2016), May 2016, Kuala Lumpur, Malaysia. 10.1109/ICC.2016.7511597 hal-01880177

\section{HAL Id: hal-01880177 \\ https://hal.science/hal-01880177}

Submitted on 16 Jul 2020

HAL is a multi-disciplinary open access archive for the deposit and dissemination of scientific research documents, whether they are published or not. The documents may come from teaching and research institutions in France or abroad, or from public or private research centers.
L'archive ouverte pluridisciplinaire HAL, est destinée au dépôt et à la diffusion de documents scientifiques de niveau recherche, publiés ou non, émanant des établissements d'enseignement et de recherche français ou étrangers, des laboratoires publics ou privés. 


\title{
WSN4QoL: WSNs for Remote Patient Monitoring in e-Health Applications
}

\author{
S. Tennina*, M. Santos*, A. Mesodiakaki ${ }^{\dagger}$, P.-V. Mekikis $^{\dagger}$, E. Kartsakli ${ }^{\dagger}$, A. Antonopoulos ${ }^{\#}$, \\ M. Di Renzo ${ }^{\ddagger}$, A. Stavridis ${ }^{\S}$, F. Graziosi*, L. Alonso ${ }^{\dagger}$ and C. Verikoukis ${ }^{\#}$ \\ ${ }^{*}$ WEST Aquila S.r.l., L'Aquila, Italy \\ \{tennina,manuel.santos,fabio.graziosi\} at westaquila.com \\ ${ }^{\dagger}$ Technical University of Catalonia (UPC), Signal Theory and Communications Barcelona, Spain \\ \{agapi.mesodiakaki, vmekikis, ellik, luisg\} at tsc.upc.edu \\ $\ddagger$ Supelec CNRS, Paris, France \\ marco.direnzo at lss.supelec.fr \\ ${ }^{\S}$ Vidavo, Thessaloniki, Greece \\ info at vidavo.eu \\ \#CTTC, Barcelona, Spain \\ \{aantonopoulos, cveri\} at cttc.es
}

\begin{abstract}
Contemporary technologies as implemented in the field of healthcare have provided the everyday clinical practice with a plethora of tools to be used in various settings. In this field, distributed and networked embedded systems, such as Wireless Sensor Networks (WSNs), are the most promising technology to achieve continuous monitoring of aged people for their own safety, without affecting their daily activities. WSN4QoL is a Marie Curie project involving academic and industrial partners from three EU countries, which aims to show how new WSNs-based technologies suit the specific requirements of pervasive healthcare applications. In particular, in this paper, the WSN4QoL's system architecture is presented as designed to exploit the Network Coding (NC) mechanisms to achieve energy efficiency in the wireless communications and distributed positioning solutions to locate patients in indoor home environments. The system has been validated through experimental activities using commercial off the shelf (COTS) WSN testbeds and medical devices prototypes offered by a commercial partner. Results demonstrate that NC helps in achieving substantial gains in terms of energy efficiency as compared to traditional relay mechanisms, while the proposed positioning solution is able to locate people in indoor environments at a sub-room accuracy level, without requiring any extra dedicated hardware.
\end{abstract}

\section{INTRODUCTION}

e-Health, according to Health Information and Management Systems Society is defined as the application of Internet and other related technologies in the healthcare industry to improve the access, efficiency, effectiveness, and quality of clinical and business processes utilized by healthcare organizations, practitioners, patients, and consumers to improve the health status of patients. Recently a sub-segment of e-Health has emerged, known as $m$-Health, where $m$ indicates the use of mobile communications, thus it extends the above definition by including the use of emerging mobile communications and network technologies for healthcare [1]. In 2010, the mHealth Summit of the Foundation of the National Institutes of Health came up with yet another definition, this time shifting the weight of implementation to the devices: the delivery of healthcare services via mobile communication devices [2].
Despite these incremental definitions, e-Health ${ }^{1}$ is not a surrogate for the clinician. It does provide the means to extend the reach of the provider beyond a face-to-face patient encounter, with the advantage of expanding the delivery of medical services when limited resources and expertise are available. For instance, using electronic images and pictures, diagnoses may be made from a remote location, either within or outside the facility. The e-Health system architecture includes several dimensions that can be materialized according to the specific environment: (i) Internet: access to information and sites with adequate user grants per category (doctors, nurses, administration, etc.) or universal access to public content (e.g., education material with epidemiological characteristics). (ii) Extranet: secure, remote connections between predefined participants (links between primary, secondary and tertiary healthcare levels) even including e-Commerce. (iii) Intranet: communications infrastructure within the enterprise, which may facilitate the access to the core data systems to all participants in the healthcare delivery chain. (iv) Core Data Systems (CDSs): function-based systems supporting the key processes of the enterprise. These may be financial, administrative or clinical systems, including the Electronic Health Record (EHR). (v) Telecommunications: the physical and technical layer enabling the connections and interchange of information through all available media, including wireless, cable and any other new and emerging means. (vi) Hardware: computers, smartphones, servers and a plethora of sensors capturing vital signs, to be used in conjunction with Diagnostic Support Systems (DSS) to provide the physical support for this infrastructure. Overall, the e-Health environment is given by the seamless interaction among these layers.

Focusing on the hardware and telecommunications fields, the rapid development of new technologies is reflected in the adoption of new cheap devices, targeting to measure and report an individual's vital signs to the CDSs or even to track his/her position in case of an emergency, as it is often the

\footnotetext{
${ }^{1}$ Here the terms e-Health and m-Health will be used interchangeably, as our focus lies in the field of wireless and mobile communications.
} 
case with elderly suffering from senile dementia. These sensors come into the form of lightweight portable (or even wearable) devices for enhanced user acceptability. The limitations, as well as the challenges for the research community, are as follows: (i) Power autonomy - the more the supported functions and the longest the time of monitoring are, the greater the energy demands will be. Increasing the batteries' size is not an option as it will lead to weightier products, drastically reducing both usability and users' satisfaction. (ii) Ubiquitous tracking of patients - currently it is based on GPS/GPRS. However, indoor operation is proven to be problematic with the aforementioned combination of technologies, while the user requirements should be strictly followed. (iii) Security - an individual's most sensitive data are connected to his/her health condition, then convincing patients to deliver such data over public or even wireless networks is one of the inhibitors of the e-Health applications.

In agreement with the above issues, the WSN4QoL project [3] involves the design of pervasive Wireless Sensor Networks (WSNs) specifically suited to meet healthcare application requirements and overcome the above mentioned limitations. This paper aims to present in a system-level perspective the new WSN4QoL's solutions, as well as an efficient protocol stack architecture to support them.

Accordingly, the remainder of the paper is organized as follows. Section II reviews some of the most relevant work related with WSN4QoL. Section III focuses on the main design challenges of a WSN-based system and the WSN4QoL's objectives, and presents the protocol stack architecture. Section IV describes the implementation of the full protocol stack designed to support such solutions and presents the results obtained through experimental activities conducted in a real home environment for remote patients' assistance. Finally, Section V concludes the paper.

\section{RELATED WORK}

In this Section our aim is to present an overview of some of the most recent and relevant projects to show which solutions are really implemented in real-world applications. A comprehensive list of projects is also available in [4].

a)HEALTH@HOME: In this project [5], a set of wearable sensors remotely collects patients' cardiovascular and respiratory parameters. Data are continuously gathered through an automatic processing system and made available to medical staff, who may take action when needed. The architecture follows the typical client/server paradigm, where the client side is a residential gateway, located at the patient's home, able to collect data from the biomedical sensors through wireless BlueTooth links. The server is installed at the health service facilities and is integrated with the Hospital Information System. The GW communicates with the server through ADSL or Cellular network using HTTPS protocol, while alarms are sent directly to the physicians as SMS.

b) $C A A L Y X-M V$ : The goal of this project [6] is to validate an innovative and efficient ICT-based solution, focused on improving the elder's quality of life by prolonging the time they can stay safer, autonomous and independently at home. The technology relies on a Mobile Subsystem, which includes a smart garment, with all sensors integrated into a Wireless
Body Area Network (WBAN) and a mobile phone. The sensor data and alarms conditions are continuously monitored and sent through standard low-cost networking equipment, namely DSL (Digital Subscriber Line) residential routers and set-topboxes when the user is at home. A standard GPS enabled smart phone (3G/UMTS) able to perform efficient network management $(3 \mathrm{G} / \mathrm{WiFi}$ switching) runs a fully autonomous software, requiring no user interaction, which manages the personalised WBAN and continuously analyses sensor data, in order to identify problem conditions and promptly alert health professionals.

c) Help4Mood: It aims at developing a system to help people with major depression recover in their own home [7]. The main components include (i) a personal monitoring system to keep track of important aspects of behaviour, such as activity levels, (ii) an interactive virtual agent asking patients about their health and well-being and providing a portal to trusted health information, and (iii) a virtual agent-based decision support system, to allow for customizations based on the individual's needs. The communication architecture [8] is based on the classical scheme, where Bluetooth is the preferred communication technology or, in some cases, the TI SimpliciTI [9] is adopted, since it is characterized by low data rate, low duty cycle, but usually longer battery life.

d) WISERBAN: WISERBAN [10] is a FP7 EU project focused on improving personal sensing capabilities by using miniature, unobtrusive, long-lifetime WBAN sensor nodes. Differently from the classical BlueTooth or ZigBee communication technologies, WISERBAN proposes a new highly integrated radio \& antenna \& data-processing microsystem built upon a heterogeneous SiP (System-in-Package) platform, addressing the miniaturized assembly of the wireless microsystem components.

e) WSN4QoL: WSN4QoL [3] is a 4-years project started in 2011. With the intent of bringing together experts, from industry and academia, it proposes the use of advanced Wireless Sensor Network (WSN) technologies for pervasive healthcare applications. In particular, it aims at providing Network Coding (NC) for multi-hop/cooperative diversity in the data communication protocol, as well as distributed localization algorithms to meet the specific requirements of WSNsenabled healthcare applications, namely, energy-efficiency, low-latency, data reliability, context-awareness and security.

To conclude, Table I provides a comparison among the main characteristics of the related work (i.e., projects) presented here. The items considered are the answers to the following questions: (i) Are security aspects addressed to preserve users' privacy? (ii) Does the project include any mechanism to track patients' position in the working environment? (iii) Besides the specific medical aspects, since in all cases some sort of battery-powered sensing equipment is present with capability of communicating through wireless links, is the energy efficiency of such communications considered to avoid draining the batteries resource too fast? Accordingly, Table I shows that our WSN4QoL is the only one addressing all the features considered and in particular people localization and energy efficiency issues, which are validated in a realistic environment with our prototypes, as reported in Section IV. 
Table I: Summary of the related work.

\begin{tabular}{|l|l|l|l|l|}
\hline \multicolumn{1}{|c|}{ Project } & \multicolumn{1}{|c|}{ Main Application } & Security & Location & $\begin{array}{c}\text { Energy } \\
\text { Efficiency }\end{array}$ \\
\hline HEALTH@HOME [5] & CVD & $\checkmark$ & $\boldsymbol{X}$ & $\boldsymbol{x}$ \\
\hline CAALYX-MV [6] & People independency & $\checkmark$ & $\checkmark$ & $\boldsymbol{X}$ \\
\hline Help4Mood [7] & People depression & $\checkmark$ & $\boldsymbol{X}$ & $\boldsymbol{X}$ \\
\hline WISERBAN [10] & Healthcare sensors & $\boldsymbol{X}$ & $\boldsymbol{X}$ & $\boldsymbol{X}$ \\
\hline WSN4QoL [3] & WSN enabled healthcare & $\checkmark$ & $\checkmark$ & $\checkmark$ \\
\hline
\end{tabular}

\section{WSN4QoL Design ChALLENGES AND System DESCRIPTION}

WSNs are distributed networked embedded systems, where each node combines sensing, computing, communication and storage capabilities. They are wireless ad-hoc networks composed of inexpensive nodes with sensing capabilities. These nodes communicate with one another by forming multi-hop networks and by maintaining connectivity in a centralized or distributed manner. The network topology is dynamic, since nodes' links may vary with nomadic and mobile devices.

Because of their wide variety of applications, it is envisioned that WSNs will become soon an integral part of our everyday lives [11]. However, although fundamental research results on WSNs theory and practice have been achieved for many different applications, healthcare scenarios pose some unique challenges and constraints. In particular, the efficient design of a WSNs-enabled pervasive healthcare system is characterized by the following intrinsic differences with respect to general-purpose WSNs design [12], [13]: (i) The devices have limited available energy resources, as they have a very small form factor. (ii) A low transmit power per node is needed to minimize interference and to cope with health concerns. (iii) The devices are located on the human body, which can be in motion. WSNs should therefore be robust against frequent changes in the network topology and channel variability. (iv) Data mostly consists of medical information, hence, high reliability and low delay/latency are required. (v) Stringent security mechanisms are required to preserve the private and confidential nature of data. ( $v i$ ) Context-awareness through cooperative localization in outdoors, indoors and mixed environments is crucial to enable a prompt reaction in case of emergency. (vii) The devices are heterogeneous and may require different resources from the network, in terms of data rates, power consumptions and reliability.

Along these lines, the main objective of WSN4QoL is to provide fundamental research advances, proof-of-concepts and real-life implementations of the enabling technologies for a winning use of WSNs-based systems in pervasive healthcare applications. More specifically, disruptive techniques such as cooperative communications, Network Coding (NC) and distributed localization algorithms are investigated. The proposed solutions are designed, optimized, and in the near future implemented in real medical devices by taking into account the very specific requirements listed above. Accordingly, the research objectives of WSN4QoL include: (i) To design a protocol stack architecture, which can accommodate a variety of protocols, algorithms, sensor devices. (ii) To develop energy-efficient and performance-guaranteed cooperative protocols and $\mathrm{NC}$ schemes. (iii) To propose advanced distributed localization protocols and algorithms specifically suited for the scenarios (e.g., indoors) targeted by the application. (iv) To conceive

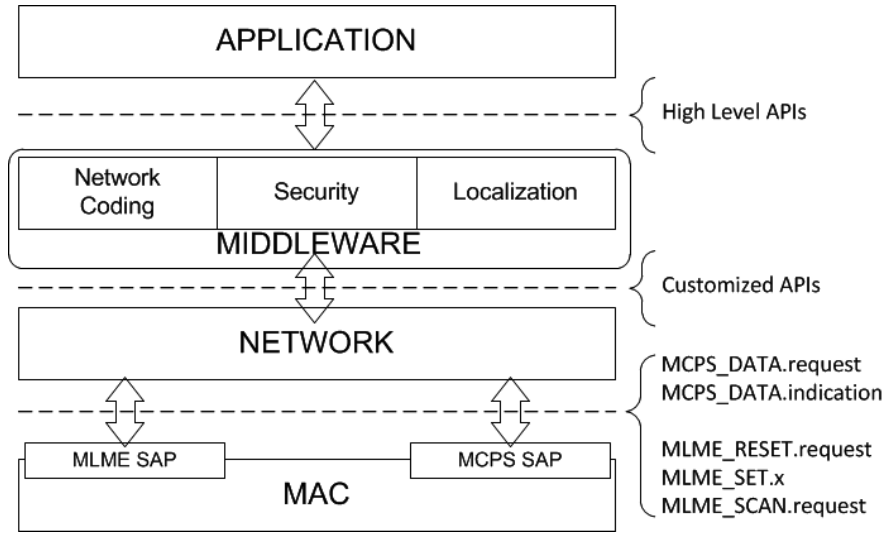

Figure 1: Communication Protocol Stack on top of the IEEE 802.15.4 standard [14]. Basic interfaces and roles of each layer are also shown.

effective, efficient, robust and resilient security solutions for the proposed algorithms and protocols. (v) To assess the performance of the proposed protocols and algorithms by means of analysis and computer simulations, by taking into account current state-of-the-art solutions and realistic requirements. (vi) To implement and assess the performance of the protocol stack in a WSN testbed. (vii) To integrate the proposed solutions in real medical devices and validate them in realworking environments.

As we proposed early in [15], the communication protocol stack can be summarized as depicted in Figure $1^{2}$. Moving from the bottom, it is composed by the following entities. (i) IEEE 802.15.4 MAC Layer: This layer is responsible for the access to the wireless medium for transmission and reception of the frames, for both mobile hub and fixed relay nodes. Among the options offered by the standard [14], we have chosen to refer to the non-beacon enabled mode, i.e., the fully asynchronous version. This choice is motivated by the fact that nodes can have variable duty cycle, especially the mobile ones. Moreover, to fully leverage on the power of NC mechanisms in terms of energy savings and network throughput gains, messages sent by the mobile nodes need to be transmitted in broadcast, without a prior association mechanism, which, on the contrary, would be necessary in the synchronous mode. (ii) Network Layer: This layer is responsible for the end-toend communication between the mobile nodes and the sink, as well as for the network's organization. The proposed solution is inspired by the ZigBee standard, thus the APIs provided

\footnotetext{
${ }^{2}$ MLME SAP stands for MAC sublayer management entity service access point, while MCPS SAP for MAC common part sublayer service access point. For further details, refer to [14].
} 
by this layer result from a customization of those defined there. In particular, the transmissions of the relay nodes in the distribution network are scheduled according to the Zigbee Cluster Tree approach, which is extensively described in [16]. (iii) Middleware Services: This layer represents an interface between the underlying protocol stack and the application layer, and is the core of the novelties introduced within the frame of WSN4QoL project, where the above mentioned algorithms are implemented. In particular, the layer is composed by three major blocks: $(a) \mathrm{NC}$; $(b)$ (Distributed) Localization, and (c) Security ${ }^{3}$. (iv) Application Layer: This last layer mainly focuses on gathering measurements from the sensors and performing data compression. For instance, at this layer the compressed sensing techniques, which leverage on the correlation of the biometric data, are implemented by using the APIs offered by the middleware. An example of that, for the case of ECG measurements, is available in [17], [18].

\section{SySTEM IMPLEMENTATION AND VALIDATION}

In order to assess the performance of the designed protocol stack, a network composed by TelosB nodes [19] (programmed with the Official TinyOS IEEE 802.15.4 implementation, as detailed in [16, Sect. 2.1], available from [20]) and real medical devices enhanced with wireless communications capabilities, prototyped by a commercial partner of WSN4QoL project, has been deployed in a real indoor environment, where, e.g., two persons can live under continuous monitoring. This environment is an apartment of around 60 square meters and composed by 6 rooms, as sketched in Figure 2. In particular, the deployed network has been used to demonstrate the performance of two of the middleware services, i.e., the network coding and the distributed localization system. To do so, the network was composed by: (a) 6 fixed TelosB nodes, deployed as one per room, playing the role of anchors for the localization system and relay for the XOR-based NC scenario and having a scheduling for their transmissions according to the scheme of [16]. (b) 2 mobile medical prototype nodes actually measure the body temperature and are carried by two persons. They move in predefined locations of the apartment. These nodes were used to broadcast the acquired data at the highest sampling speed to the destination or through the relay nodes. Every time a person is steady in a predefined location, nodes also update their position estimation. To prove the gains introduced by our localization solution (the Enhanced Steepest Descent, ESD) against the classical steepest descent (SD), one node has been programmed to run the former while the other runs the latter algorithm. (c) 1 fixed TelosB destination (playing the role of the $\mathrm{GW}$ ) is linked to a PC and was placed in the living room and used to collect measurements coming from the nodes and record the statistics for later analysis. Although the best position for the GW should be the center of the network, in order to assess the performance in more generic and realistic conditions, we had to place it at the apartment's main entrance, i.e., near a wired Internet plug.

\footnotetext{
${ }^{3}$ This module is responsible for monitoring the acknowledgement packets exchanged at the network layer among the nodes, searching for potential threats or nodes malfunctioning situations. However, due to space constraints, the details of this module are not shown in this paper.
}

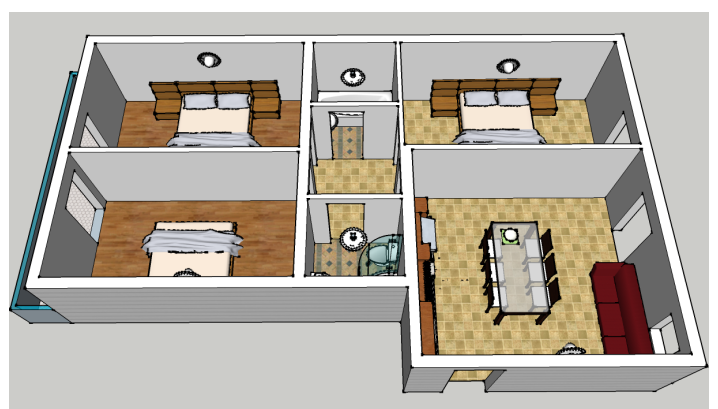

Figure 2: Indoor Environment where the WSN is deployed.

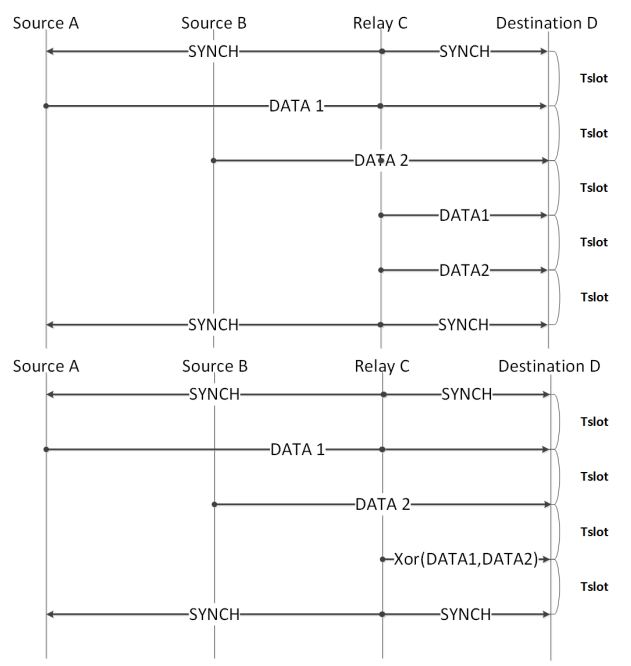

Figure 3: Timings of the Multi Access Relay Channel scenario. [top] Baseline scenario: relay forwards two messages. [bottom] Network Coding: relay broadcasts a single message.

\section{A. Support for Network Coding}

Given the position of the GW, the resulting network between the GW and the fixed relay/anchor nodes has a clustertree-like topology [16], where the GW is at depth 0 and the maximum depth is 3 (i.e., the farthest node reaches the destination in up to 3 hops). The transmissions are scheduled according to [16] in order to avoid inter-nodes mutual interferences. However, the distances among the nodes allow to exploit overhearing, which is used to generate the necessary redundancy of the packets at the relay nodes. The latter ones can opt for (a) discarding (non-cooperative mode), (b) relaying (cooperative relay only mode) or (c) relaying with NC XORbased combination (cooperative relay with $\mathrm{NC}$ mode) the intercepted packets.

To simplify the description of the NC solution implemented at the relay nodes, we present the case of a single relay network. This scenario is often referred to as Multi Access (Single) Relay Channel and is illustrated next. Two sources A and $\mathrm{B}$ are mobile nodes carried by two patients, a relay node $\mathrm{C}$ has a fixed position and an extra node $\mathrm{D}$ is the destination of the measurements reports. Figure 3 presents the timings characterizing the considered scenario. In particular, the relay $\mathrm{C}$ is responsible of defining the network scheduling among 

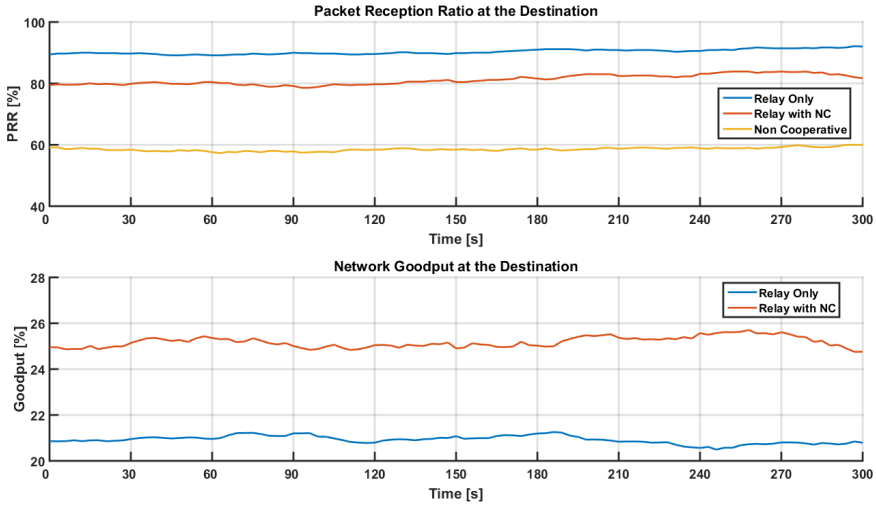

Figure 4: Average Network Performance. [Top] Packet Reception Ratio. [Bottom] Goodput. The values shown represents the average every 2 seconds interval.

the source nodes A and B by broadcasting synch packets. The source nodes are programmed to send a message to the destination in their appropriate time slots, i.e., node A sends its data after Tslot and node B sends its data after $2 * T$ slot from the synch reception. In the baseline scenario, node $\mathrm{C}$ forwards the intercepted data from A to the destination after Tslot and data from B after another Tslot, then it broadcasts a new synch packet and the process is iterated. In the NC scenario, instead of forwarding the two messages, node $\mathrm{C}$ transmits to the destination a single message, which is the combination of the two messages from A and B based on a binary XOR operation. The XOR guarantees the property that, if just one packet is lost at the destination, let it be $D A T A 1, \mathrm{D}$ can recover it by simply computing $D A T A 1=X O R(p k t C, D A T A 2)$, where $p k t C$ is the packet received from node $\mathrm{C}$. The main advantage of $\mathrm{NC}$ against the baseline scenario is that the relay node forwards a single packet instead of two, resulting in larger energy savings and higher network throughput, since the synch message can be sent every $4 *$ Tslot instead of $5 *$ Tslot.

Figure 4 shows the network performances measured at the destination in terms of packet reception ratio and goodput (i.e., the fraction of information reaching the destination in the unit of time) in the three different behaviors at the relay nodes, as mentioned above. As it is evident, the cooperation of the intermediate nodes playing as relays is paramount to increase the overall packet reception ratio at the destination by an amount ranging from $20 \%$ to $30 \%$. While the use of NC at the relay nodes does not show major advantages in terms of packet loss as compared with the relay only case, the NC outperforms the relay when the comparison is extended to the goodput metric (Figure 4(bottom)): in this case it is evident that NC helps in achieving gains of more than 5\% in terms of goodput. Overall, we can conclude that while the presence of relays is paramount to increase the packet reception ratio, even the simple XOR-based combination of packets at the relays guarantees gains in terms of network efficiency.

\section{B. Support for Localization}

The localization algorithm adopted in WSN4QoL project has been previously described in [21]. It is an anchor-based and range-based solution, since the mobile node needs to estimate the distance from the anchors available. To do so, one solution is to adopt a ranging model based on the Received Signal Strength (RSS) measurements, i.e., a formulation which correlates the distance to the received power of the signal. Typically, this model is expressed as a function of two parameters as follows: $d=10^{\frac{R S S-A}{10 n}}$ where $d$ denotes the transmitterto-receiver distance, $n$ is the propagation path-loss exponent, $A$ is the RSS reference value measured by a receiver located at a distance $d_{0}=1 \mathrm{~m}$ from the transmitter, and RSS is the actual measured value. In order to use this model, the values of the parameters $A$ and $n$ must be chosen, through, e.g., a preliminary calibration phase in the environment.

However, in the considered applications, these coefficients are strongly dependent on the environment and usually show big fluctuations due to, e.g., signal reflections, interference and people moving around. This suggests that using any fixed and outdated estimates for these channel parameters certainly yields to less accurate distance computations and so final position estimation. To cope with this problem, our solution is to allow the anchors to exploit the synch messages (Figure 3) to carry inter-anchors RSS measurements in the IEEE 802.15.4 data frames. Since the position of the anchors are known, these updated measurements help every mobile node to estimate the actual propagation conditions, so reducing the ranging error.

To demonstrate the improvement of the ESD algorithm with this adaptive ranging model against the classical SD with fixed path loss coefficients the same setup described above has been adopted, with the only difference that, in terms of localization algorithm at the Middleware layer, one of the two mobile nodes is programmed with the ESD and the other with SD algorithms. The performance gains of the proposed ESD algorithm over the classical SD are shown in Figure 5. More in details, Figure 5(top) shows the localization accuracy of several estimations in different positions of the mobile nodes, while Figure 5(bottom) presents the convergence speed of the two algorithms. As it is clear, regardless of the specific position of the mobile nodes in the apartment of Figure 2, the ESD with the dynamic ranging parameter estimations outperforms the classical SD in terms of accuracy and number of iterations needed to converge to the final estimation. Overall, the average localization error of the ESD solution over the area of the apartment is below $2.5 \mathrm{~m}$ : this results in a roomlevel accuracy, which matches the expected requirements for e-Health applications. Nevertheless, there exist few critical situations on the borders of the area, where we were not able to acquire the position estimation, due to the lack of the information from the minimum number of anchors (i.e., in some border locations some anchors were hidden to the mobile nodes). This issue can be solved by improving the coverage of the anchor nodes. Along this line, radio propagation simulation softwares (e.g., [22]) could provide indications about the optimal anchors' number and positions.

\section{Conclusion}

Pervasive and mobile e-Health applications are becoming critical in everyone's life. In our WSN4QoL project, we aim to propose a WSN-based system architecture to allocate enhanced solutions to achieve energy efficiency in wireless communications and patients' localization in indoor environments, while meeting the overall application requirements. In this paper 

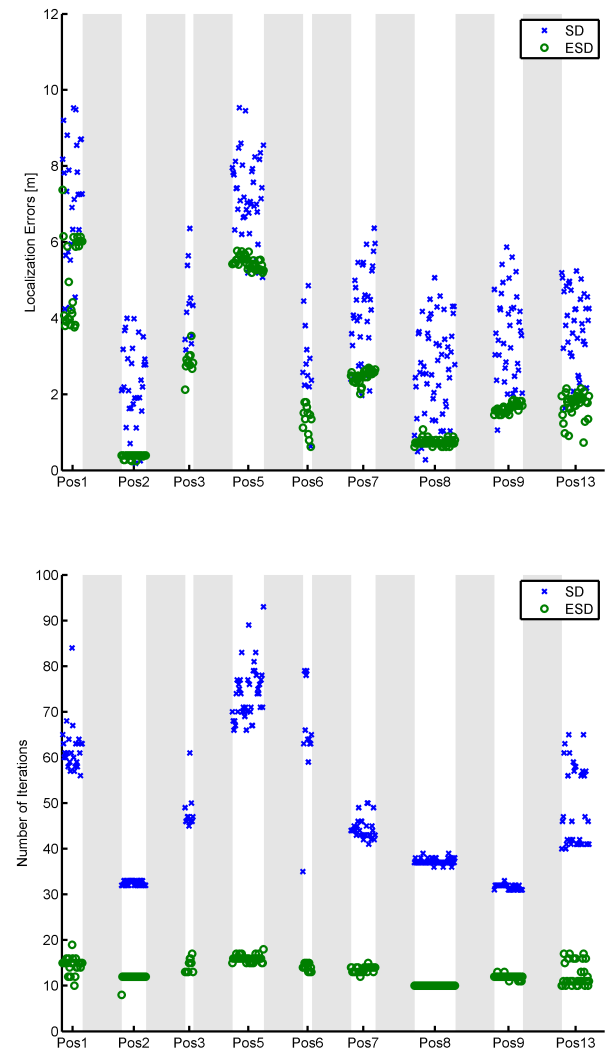

Figure 5: Accuracy of the localization methods and convergence speed for sample positions in the apartment of Figure 2. [Top] Localization Error. [Bottom] Convergence Speed.

we presented the novel solutions designed in the frame of this project and proposed the protocol stack to support them. A proper validation of the algorithms has been carried out through the implementation over a real WSN.

Our results have demonstrated the efficacy of the proposed system for addressing applications where patients are in a relatively small area, such as in their home. In this case, the coverage of the wireless network is guaranteed through relay nodes over a rather limited number of hops. The application in larger scale environments (e.g., hospitals) is an on-going research activity, which can move from the consideration that any large scale network might be efficiently decomposed into smaller scale WSNs rooted at appropriate gateways, interfacing them with an existing infrastructure (e.g., WLAN), to form a hierarchical network architecture. We expect that the energy efficiency achieved by our solution will keep low the requirement on the battery capacity, so that the weight of the final device can be kept low as well, and this will increase the chances that people will effectively accept and use it.

\section{ACKNOWLEDGMENT}

This research was supported by the European Commission under the Marie Curie IAPP WSN4QoL project, grant no. IAPP-GA-2011-286047.

\section{REFERENCES}

[1] R. Istepanian, S. Laxminarayan, and C. S. Pattichis, M-health. Springer, 2006.

[2] C. Torgan. (2009, Nov) The mHealth summit: Local \& global converge. [Online]. Available: www.caroltorgan.com/mhealth-summit

[3] WSN4QoL. (2015) Wireless sensor networks for quality of life. [Online]. Available: www.wsn4qol.eu

[4] CompAAL. (2012, April) AAL projects in EU. Web Wiki. [Online]. Available: http://eeyempilot.eap.gr/compaal_wiki/index.php/ AAL_projects_in_EU

[5] L. Fanucci. (2014) Health at home. [Online]. Available: www. aal-europe.eu/projects/healthhome

[6] CAALYX-MV. (2011) Complete ambient assisted living experiment market validation. [Online]. Available: www.caalyx-mv.eu

[7] Help4Mood. (2014) A computational distributed system to support the treatment of patients with major depression. [Online]. Available: www.help4mood.info/site/default.aspx

[8] D. Perez-Diaz de Cerio, S. Ruiz Boque, J. Rosell-Ferrer, J. RamosCastro, and J. Castro, "A wireless sensor network design for the Help4Mood european project," European Wireless, 2012. EW. 18th European Wireless Conference, pp. 1 -6, april 2012.

[9] Texas Instruments. SimpliciTI compliant protocol stack. [Online]. Available: www.ti.com/tool/simpliciti

[10] WISERBAN. (2014) Smart miniature low-power wireless microsystem for body area networks. [Online]. Available: www.wiserban.eu

[11] P. Harrop and R. Das, "Wireless sensor networks (WSN) 2012-2022: Forecasts, technologies, players - the new market for ubiquitous sensor networks (USN)," IDTechEx, Tech. Rep., Decermber 2012.

[12] H. Alemdar and C. Ersoy, "Wireless sensor networks for healthcare: A survey," Comput. Netw., vol. 54, no. 15, pp. 2688-2710, Oct. 2010. [Online]. Available: http://dx.doi.org/10.1016/j.comnet.2010.05.003

[13] S. Ullah, H. Higgins, B. Braem, B. Latre, C. Blondia, I. Moerman, S. Saleem, Z. Rahman, and K. S. Kwak, "A comprehensive survey of wireless body area networks," J. Med. Syst., vol. 36, no. 3, pp. 1065-1094, Jun. 2012. [Online]. Available: http: //dx.doi.org/10.1007/s10916-010-9571-3

[14] IEEE, "Standard for information technology - local and metropolitan area networks - specific requirements - part 15.4: Wireless medium access control (MAC) and physical layer (PHY) specifications for low rate wireless personal area networks (WPANs)," IEEE Std 802.15.42006 (Revision of IEEE Std 802.15.4-2003), pp. 1-320, Sep. 2006.

[15] S. Tennina, M. Di Renzo, E. Kartsakli, F. Graziosi, A. Lalos, A. Antonopoulos, P. V. Mekikis, and L. Alonso, "WSN4QoL: a WSNoriented healthcare system architecture," International Journal of Distributed Sensor Networks, vol. 2014, 2014.

[16] S. Tennina, A. Koubaa, R. Daidone, M. Alves, P. Jurcik, R. Severino, M. Tiloca, J.-H. Hauer, N. Pereira, G. Dini, M. Bouroche, and E. Tovar, IEEE 802.15.4 and ZigBee as Enabling Technologies for Low-Power Wireless Systems with Quality-of-Service Constraints. SpringerBriefs in Electrical and Computer Engineering, 2013, p. 464, iSBN: 978-3642-37367-1

[17] A. S. Lalos, L. Alonso, and C. Verikoukis, "Model based compressed sensing reconstruction algorithms for ECG telemonitoring in WBANs," Digital Signal Processing (ELSEVIER), 2014.

[18] A. Lalos, A. Antonopoulos, E. Kartsakli, M. Di Renzo, S. Tennina, L. Alonso, and C. Verikoukis, "RLNC-aided cooperative compressed sensing for energy efficient vital signal telemonitoring," Wireless Communications, IEEE Transactions on, vol. 14, no. 7, pp. 3685-3699, July 2015

[19] MEMSIC. (2015) TelosB mote platform. MEMSIC Inc. [Online]. Available: www.memsic.com

[20] TinyOS. (2014) Main development repository. [Online]. Available: https://github.com/tinyos/tinyos-main

[21] S. Tennina, M. Di Renzo, E. Kartsakli, F. Graziosi, A. Lalos, A. Antonopoulos, V. Mekikis, and L. Alonso, "WSN4QoL: A WSNoriented healthcare system architecture," International Journal of Distributed Sensor Networks, vol. 2014, p. 16, 2014.

[22] AWE. (2013) WinProp software package. AWE Communications. [Online]. Available: www.awe-communications.com 